\title{
In Vivo Immunological Effects of CD73 Deficiency
}

\author{
Thomas Adam ${ }^{\mathrm{a}} \quad$ Andreas Mathes ${ }^{\mathrm{a}, \mathrm{b}} \quad$ Orkhan Isayeva,c $^{\mathrm{a}, \mathrm{Cavel}}$ P. Philippov ${ }^{\mathrm{d}}$ \\ Jens Werner ${ }^{a, e}$ Svetlana Karakhanova ${ }^{f, g} \quad$ Alexandr V. Bazhin ${ }^{a, e}$ \\ aDepartment of General, Visceral, and Transplant Surgery, Ludwig-Maximilians-University Munich, \\ Munich, Germany, 'Sana Klinikum Offenbach, Offenbach, Germany, 'Department of Histology, \\ Embryology and Cytology, Azerbaijan Medical University, Baku, Azerbaijan, 'Department of Cell \\ Signalling, Belozersky Institute of Physico-Chemical Biology, Lomonosov Moscow State University, \\ Moscow, Russia, e'German Cancer Consortium (DKTK), Partner Site Munich, Munich, Germany, \\ 'Department of General, Visceral and Transplantation Surgery, University of Heidelberg, Heidelberg, \\ Germany, ${ }^{9}$ Section Surgical Research, University of Heidelberg, Heidelberg, Germany
}

\section{Key Words}

$\mathrm{Cd} 73 \cdot \mathrm{Immune}$ regulation $\bullet \mathrm{Immune}$ regulatory molecules $\bullet$ Knockout mice

\begin{abstract}
Background/Aims: The extracellular ecto-5'-nucleotidase (CD73) is involved in the production of immunosuppressive adenosin (Ado), which can influence different immune cells through the specific adenosine receptors. The main aim of this work was to characterize immune cell populations as well as serum cytokine level in healthy CD73-deficient mice compared to healthy wild-type animals. Methods: Profound immnophenotyping of splenocytes from healthy CD73-deficient and wild-type mice was done using flow cytometry (FACS analysis). Cytokine measurement in the serum of the animals was carried out with a Bio-Plex assay. Results: The CD73-deficience leads to an increase in a percentage of NK cells and pDC, as well as influences expression of the costimulatory molecules CD80 and CD86. The knockout mice in opposite to wild-type animals show high amount of effector CD4+ $\mathrm{T}$-cells in the spleens. No changes have been found in the subpopulations of CD8 ${ }^{+} T$-cells. Besides, CD73-deficience leads to a decrease in the percentage of regulatory $T$ cells. Compared with the wild-type animals we found that CD73 knockout mice possess low serum concentration of IL-6. Conclusion: This in vivo study clear demonstrated certain immunological changes in the CD73-deficient mice and thus immunoregulatory potential of CD73 molecule. This makes this extracellular enzyme to a real immune check point molecule, attractive for further investigations and clinical studies.

S. Karakhanova and A. V. Bazhin contributed equally to this work.

\begin{tabular}{ll}
\hline A.V. Bazhin & Department of General, Visceral, and Transplant Surgery, Ludwig-Maximilians-University Munich \\
& Marchioninistr. 15, 81377 Munich (Germany) \\
& Tel. +49-89-4400-0, Fax +49-89-4400-76433 E-Mail alexandr.bazhin@med.uni-muenchen.de
\end{tabular}
\end{abstract}




\section{Cellular Physiology Cell Physiol Biochem 2019;52:1193-1202 \\ \begin{tabular}{c|c|c|c|}
\hline DOl: 10.33594/000000081 & O 2019 The Author(s). Published by \\
\hline
\end{tabular} \\ \begin{tabular}{l|l} 
Published online: 19 April 2019 & Cell Physiol Biochem Press GmbH\&Co. KG \\
\hline
\end{tabular} \\ Adam et al.: Immunological Effects of CD73 Deficiency}

\section{Introduction}

For the faultless function of the immune system, tight regulation of immune cell activation, immuno-suppression and the strength and efficiency of the immune response is essential. Mechanisms of immune regulation ensure that pathogens can be effectively combated while preventing the induction of autoimmunity and tissue damage. One of such mechanism could be represented by the adenine nucleoside - adenosine (Ado). In general, intracellular Ado level reflects the cellular oxygen phosphorylation and represents the intermediate metabolite of the ATP catabolism [1]. In case of extracellular ATP, this molecule undergoes catabolic processing by a co-play of two enzymes: first the extracellular ectonucleoside triphosphate diphosphohydrolase-1, better known as CD39, hydrolyzes ATP to ADP which in turn serves as a substrate for the extracellular ecto-5'-nucleotidase (CD73) leading to Ado production [2]. The enzyme consists of a homodimer placed in the cellular membrane by glycophosphatidylinositol anchors [3]. Throughout healthy tissues, CD73 is widely expressed: in epithelial cells of the respiratory tract, smooth muscle cells, cardiac myocytes and other tissues. Expression of CD73 subjects to controlling by different transcriptional factors (i.e. SP1, AP-2, SMAD) and by microRNA [4].

Ado has a very short half-life and it is catabolized in few seconds in the inert inosine by a membrane-bound adenosine deaminase [5]. However, in this short life-time Ado can commit important functional activity. Ado interacts with four distinct cell-surface G-proteinlinked receptors: A1, A2A, A2B, and A3 [6]. A1 and A3 adenosine receptors are coupled to the $G_{i / o}$ subunit, and their activation leads to the inhibition of adenylate cyclase which in turn reduces cAMP production, and as a consequence inhibits protein kinase A activation. Activation of $\mathrm{A} 2 \mathrm{~A}$ and $\mathrm{A} 2 \mathrm{~B}$ receptors has been also reported to belong to immunosuppressive effects of Addo [7]. A2A receptors are expressed on a variety of immune cells, including T lymphocytes, NK cells, DC, macrophages, and granulocytes [8] making these immune cells sensitive to Ado. Besides, immune suppressive cells as T regulatory cells (Treg) produced Ado inhibiting the activity of various immune cell subpopulations [9]. In context of tumorinduced immune suppression, Ado can be produced by myeloid-derived suppressor cell (MDSC) therefore making Ado to one of inhibiting mechanisms of MDSC [10]. This evidence allows us to assume that CD73 represents a real immune check point molecule [11].

CD73 knock-down (CD73KO) mice were created by Linda Thompson and deeply examined them in relation to endothelial permeability [12]. Homogenous mice are viable and fertile and they are frequently used for investigation of vascular permeability. While being used as a model in cancer research, the native CD73KO mice are little investigated in the immunological context. Therefore, we aimed to profoundly analyze immune cell phenotypes as well as cytokine profiles in the healthy CD73KO mice.

\section{Materials and Methods}

\section{Materials}

The antibodies used for the flow cytometry analyses (FACS) are listed in the Supplementary Table 1 (for all supplemental material see www.cellphysiolbiochem.com), purchased from BD Biosciences (Germany). Luminex kits to analyze cytokine production were purchased from Bio-Rad Laboratories GmbH (Germany). PCR Kits for genotyping of new born CD73 mice were purchased from Bioline, Invitrogen and Eurofins Genomics (Germany).

\section{Mice}

CD73KO mice (B6.129S1-Nt5etm1Lft/J) in BL/6 background were originally designed by Thompson et al [12]. and were purchased by Jackson Lab (Germany). The mice were crossed and kept under specific pathogen-free conditions in the animal facility of the Center of Neuropathology and Prion Research of the LMU Munich. Homozygous CD73KO mice were routinely checked for the KO genotype stability. C57BL/6 (Bl6) mice were purchased by Charles River (Germany). Animal experiments were carried out after approval 


\section{Cellular Physiology Cell Physiol Biochem 2019;52:1193-1202

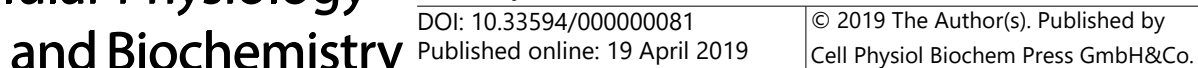 \\ Adam et al.: Immunological Effects of CD73 Deficiency}

by the authorities (Regierungspraesidium Oberbayern, 55.2-1-54-2532-195-2014). Bl6 and CD73KO mice at the age of 8-12 weeks were used for the study. Blood was collected under anesthesia by the cardiac puncher; afterwards the mice were sacrificed and their spleens removed and used for preparation of singlecell splenocyte suspensions [13].

\section{Flow cytometry analysis of murine splenocytes}

Flow cytometry (FACS) analysis of murine splenocytes was performed as described previously [14]. Freshly isolated spleen cell suspension was resuspended in the stain buffer (PBS supplemented with $1 \%$ mouse serum and $1 \mathrm{mM}$ EDTA). The cells were counted and the density was adjusted to $5 \times 10^{6} / \mathrm{ml}$. Then, $100 \mu \mathrm{l}$ of cell suspension $\left(5 \times 10^{5}\right.$ cells) was incubated with various monoclonal antibodies at room temperature in the dark for $30 \mathrm{~min}$. The amount of each antibody had been titrated before the experiments. After washing and rewashing with the stain buffer, the cells were resuspended in $100 \mu \mathrm{l}$ of the stain buffer and transferred to a $5 \mathrm{ml}$ polystyrene tube containing $300 \mu \mathrm{l}$ of the stain buffer for flow cytometry analysis. The Foxp3 staining buffer set (EBioscience, Germany) was used for intracellular staining, according to the manufacturer's instructions.

Several panels with different combinations of antibodies were used in our experiments to identify specific cell subpopulations and to investigate their activation status: (1) panel for $\mathrm{CD}^{+}{ }^{+}$and $\mathrm{CD} 8^{+}{ }^{\mathrm{T}}$-cells $\left(\mathrm{CD}^{+}\right.$cells), as well as for NK (CD3-NK1.1 $1^{+}$) and NKT (CD3 $\left.{ }^{+} \mathrm{NK} 1.1^{+}\right)$cells. CD69 was a marker for activated NK and NKT cells. Different subsets of $\mathrm{CD} 4^{+}$and $\mathrm{CD} 8^{+} \mathrm{T}$-cells were characterized as naïve T-cells (CD62 $\mathrm{L}^{+} \mathrm{CD} 44^{-}$), effector T-cells (CD62 $\mathrm{L}^{-} \mathrm{CD} 44^{-}$) and memory T-cells ( $\mathrm{T}_{\mathrm{M}^{\prime}}$ CD62L-CD44+); (2) panel for B cells (CD3-B19+); (3) panel for Treg, which were characterized as Foxp $3^{+} \mathrm{CD} 25^{+}$in the $\mathrm{CD} 4^{+} \mathrm{T}$-cells population, Tcon were defined as $\mathrm{CD}^{+}{ }^{+} \mathrm{FoxP}^{-} \mathrm{CD}^{-} 5^{-}$cells and activated Tcon were $\mathrm{CD}^{+}{ }^{+} \mathrm{FoxP}^{-} \mathrm{CD}^{-} 5^{+} ;(4)$ panel for dendritic cells (DC) as well as for myeloid cells - conventional dendritic cells (cDC, CD11 $\left.{ }^{\text {hi }} C D 11 b^{+}\right)$and plasmacytoid dendritic cells (pDC, CD11 $\mathrm{c}^{+} \mathrm{CD} 45 \mathrm{R}^{+}$) were further characterized by the expression of CD80 (B7-1) and CD86 (B7-2); PMN-MDSC were defined as CD11 ${ }^{+}{ }^{+}{ }^{2} 6 \mathrm{G}^{+} \mathrm{Ly} 6 \mathrm{C}^{\text {low }}$ and M-MDSC as CD11 $\mathrm{b}^{+} \mathrm{Ly} 6 \mathrm{G}-\mathrm{Ly} 6 \mathrm{C}^{\text {high }}$ and macrophages as $\mathrm{CD} 11 \mathrm{~b}^{+} \mathrm{Ly} 6 \mathrm{G}-\mathrm{F} 4 / 80^{+}$. All the gates were set according to the corresponding fluorescence minus one (FMO) control. Gating strategies are presented in the Fig. S1. FACSDIVA software (BD Biosciences, Germany) was used to analyze the data.

Cytokine measurement with Luminex assay

The cytokines were determined using a Bio-Plex assay [15]. The fluorescence measurement was performed using Luminex ${ }^{\circledR} 100$ System (Bio-Rad, Germany). According to the standard curves, the concentration of the respective cytokines was calculated and presented in $\mathrm{pg} / \mathrm{ml}$.

\section{Statistical analysis}

All statistical analyses were performed using GraphPad Prism Version 7.01. Distributions of continuous variables were described by median, $25 \%$ and $75 \%$ percentiles, min and max, and were presented as scatter plots for FACS data and column bar graph for Luminex data. The null hypothesis (mean values are equal) versus the alternative hypothesis (mean values are not equal) was tested for two groups by unpaired twotailed $t$-tests for normal distributed variants.

\section{Results}

The immunological effects of CD73 deficiency in vivo were carried out by the immunophenotyping of freshly isolated splenocytes from healthy wild type Bl6 and CD73 knock-down (CD73KO) mice using FACS and analyzing of serum cytokines by the Luminex approach. The percentage and phenotype of the following immune cell subpopulations, as a part of innate and adaptive immunity: NK and NKT, macrophages, CD $11 b^{+}$Ly $6 G^{+/}-L y 6 C^{\text {high }}$ / Ly6Clow, DC, B-cells, CD4, CD8, Tcon and Treg cells were assessed. The gating strategy for panel analysis is presented in Fig. S1. 


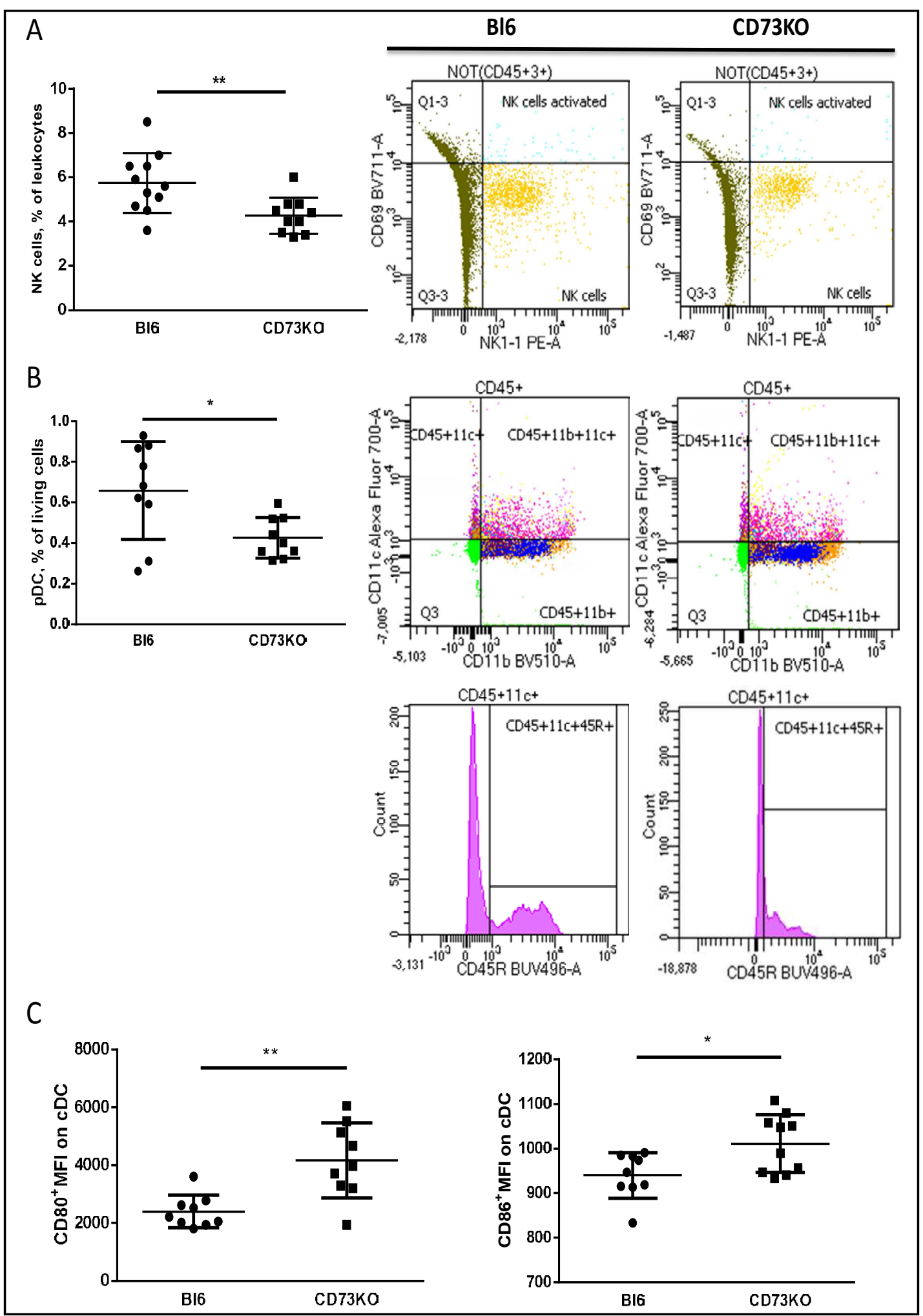

Fig. 1. Influence of CD73-deficience on the cells of innate immune system. NK cells (A) and pDC (B) in spleens of Bl6 and CD73KO mice, presented as a scatter-plot with mean and SD and analyzed with the unpaired $\mathrm{T}$ test $\left({ }^{* *} \mathrm{p}<0.01\right.$ ) - left panel. Right panel - representative FACS pictures from Bl6 vs. CD73KO mice. (C) Expression in MFI of CD80 and CD86 on the surface of cDC from spleens of Bl6 and CD73KO mice, presented as a scatter-plot with mean and SD and analyzed with the unpaired T test $\left({ }^{*} \mathrm{p}<0.05\right.$ and $\left.{ }^{* *} \mathrm{p}<0.01\right)$. 
CD73 absence leads to a low amount of NK cells and $p D C$, and influences the expression of CD80 and CD86

We first analyzed NK as well as NKT cell populations in CD73KO and Bl6 mice which were defined based on their surface markers as described in the 'Material and Methods' section. CD73KO mice showed a decrease in the percentage of NK cells $(5.75 \pm 0.41 \%$ for WT and $4.27 \pm 0.26 \%$ for CD73KO, $p=0.008$, Fig. $1 \mathrm{~A}$ ). The activation status of NK cells was not affected. No changes in the percentage of NKT cells as well as their activation state have been detected (data not shown).

Regarding DCs we sow a decrease in the frequency of pDC $(0.66 \pm 0.24 \%$ for WT and $0.43 \pm 0.1 \%$ for CD73KO, $p=0.017$ ) in the spleen of CD73KO mice (Fig. 1B), but not of cDC (data not shown). Moreover, the CD80 and CD86 expression level measured in MFI (mean fluorescence intensity) was increased in the CD73KO mice ( $p=0.0017$ for CD80 MFI and $p$ $=0.017$ for CD86 MFI, Fig. 1C). In respect of the myeloid leukocyte populations including MDSC and macrophages, we did not see any differences between Bl6 and CD73KO mice (data not shown).

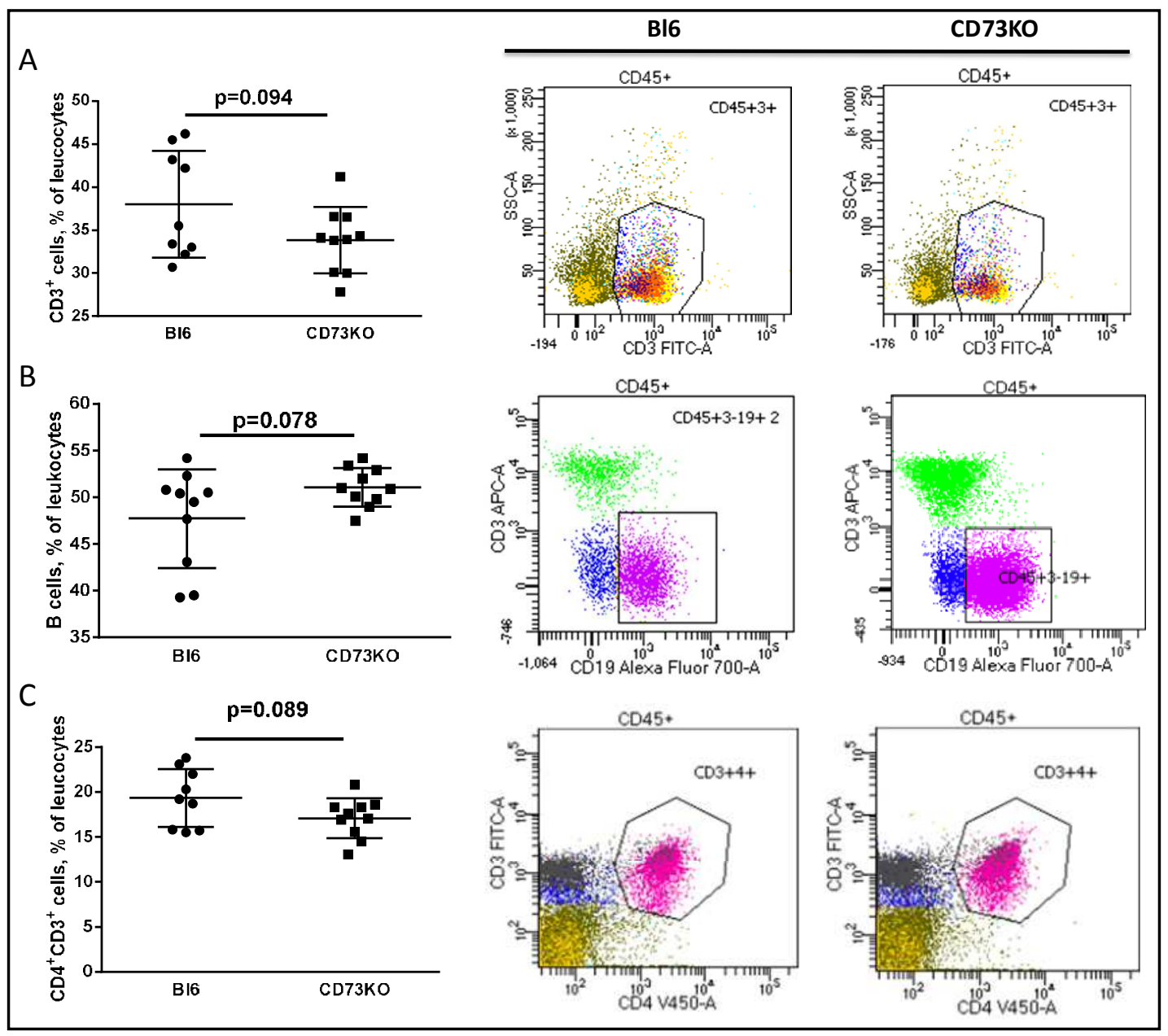

Fig. 2. Influence of CD73-deficience on the different lymphocyte populations. Lymphocytes (A), B cells (B) and $\mathrm{CD}^{+} \mathrm{T}$ cells $(\mathrm{C})$ in spleens of $\mathrm{Bl} 6$ and CD73KO mice, presented as a scatter-plot with mean and SD and analyzed with the unpaired T test - left panel. Right panel - representative FACS pictures from Bl6 vs. CD73KO mice. 


\section{CD73 deficiency promotes accumulation of effector $C D 4^{+}$T cells and reduction of the Treg} amount

First, we detected in the spleens of CD73KO mice a clear trend in a decrease in percent of immune cells in the lymphocyte gate $(37.99 \pm 6.2 \%$ for WT and $33.83 \pm 3.86 \%$ for CD73KO, $p$ $=0.09$, Fig. $2 \mathrm{~A}$ ). Also a trend to higher amount of $\mathrm{B}$ cells was found in the spleens of transgenic mice $(47.73 \pm 5.27 \%$ for WT and $51.08 \pm 2.08 \%$ for CD73KO, $p=0.078$, Fig. $2 \mathrm{~B})$. In respect of $\mathrm{T}$ cells, we sow a trend in a decrease in amount of CD4 $4^{+} \mathrm{T}$ cells $(19.34 \pm 3.22 \%$ for WT and $17.08 \pm 2.21 \%$ for CD73KO, $p=0.089$, Fig. $2 \mathrm{C}$ ). In the same time no changes have been seen either in the gate of CD8 ${ }^{+} \mathrm{T}$ cells or in their subpopulation (data not shown).

Next, we analyzed the subpopulation of T cells in-depth. The CD73KO mice demonstrated a decrease in a frequency of memory subpopulation of CD $4^{+} \mathrm{T}$ cells $(13.07 \pm 4.46 \%$ for WT and $9.35 \pm 2.15 \%$ for CD73KO, $p=0.085$, Fig. 3 ), whereat the frequency of effector subpopulation of these $\mathrm{T}$ cells was increased $(13.07 \pm 4.46 \%$ for $\mathrm{WT}$ and $9.35 \pm 2.15 \%$ for CD73KO, $p=$ 0.001, Fig. 3).

It is well-known that CD73 can be involved in the Treg-induced immunosuppression [9], therefore we were curious to explore the percentage of Treg and Tcon (conventional T-cells) in CD73KO compared to WT mice. Indeed, CD73KO mice showed a decreased percentage of Treg (8.03 $\pm 1.1 \%$ for WT and $5.76 \pm 1.1 \%$ for CD73KO, $p=0.0003$, Fig. 4$)$ as well as of activated Tcon $(2.4 \pm 0.51 \%$ for WT and $1.72 \pm 0.77 \%$ for CD73KO, $p=0.032$, Fig. 4$)$ and an increased amount of Tcon (77.93 $\pm 3.53 \%$ for WT and $82.04 \pm 2.47 \%$ for CD73KO, $p=0.007$, Fig. 4).

\section{CD73 deficiency results in the reduction of IL-6 cytokine production}

Since we observed some immunological changes in cell populations caused by the absence of CD73 molecules, we aimed to compare the serum concentrations of IL-1 $\beta$, IL-2, IL-6, IL-10, IL-13, IL-17, IFN $\gamma$, VEGF and TNF- $\alpha$ in Bl6 and CD73KO mice. Indeed, the level of IL-6 $(7.5 \pm 2.76 \mathrm{pg} / \mathrm{ml}$ for Bl6 and $5.12 \pm 1.55 \mathrm{pg} / \mathrm{ml}$ for CD73KO, $p=0.041)$ cytokine was reduced in the serum of transgenic mice. Other cytokines measured had the same serum level in both strains of mice (data not shown).

Fig. 3. Influence of CD73deficience on the different subpopulations of $\mathrm{CD} 4^{+} \mathrm{T}$ cells in spleens of $\mathrm{Bl} 6$ and $\mathrm{CD} 73 \mathrm{KO}$ mice. Data are presented as a scatter-plot with mean and SD and analyzed with the unpaired $\mathrm{T}$ test $\left({ }^{*} \mathrm{p}<0.05\right.$ and $* * * \quad \mathrm{p}<0.005$ ) - upper panel. Bottom panel - representative FACS pictures from $\mathrm{Bl} 6$ vs. CD73KO mice.

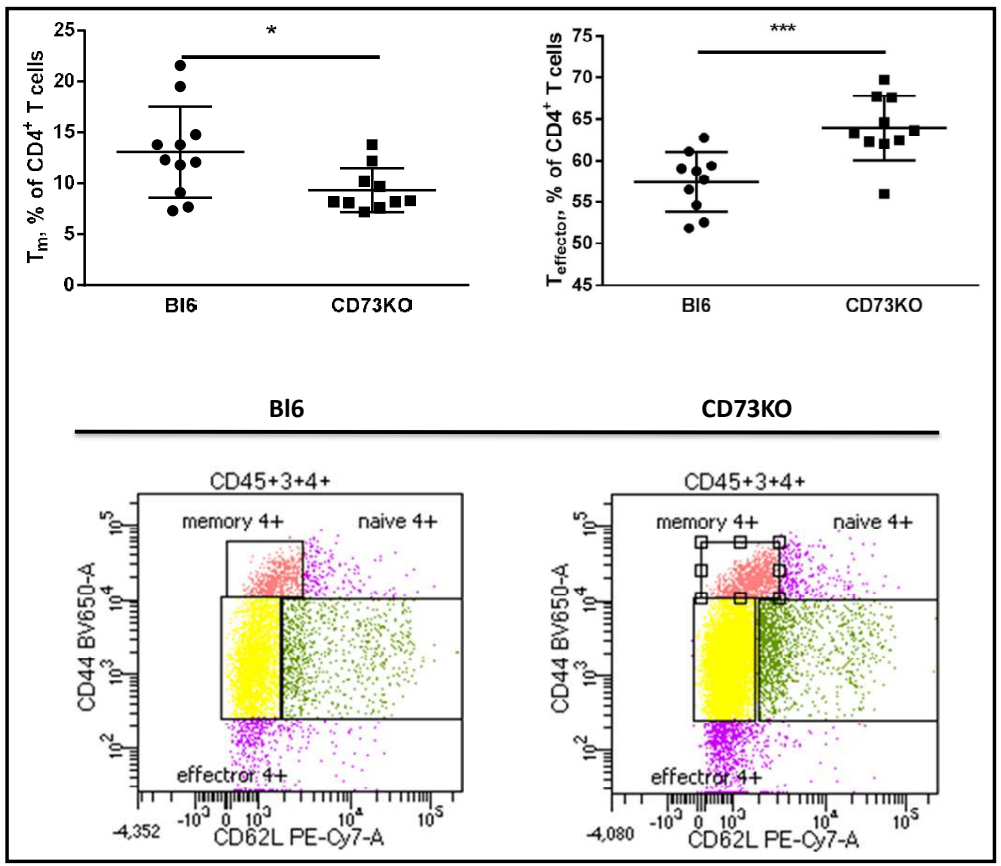




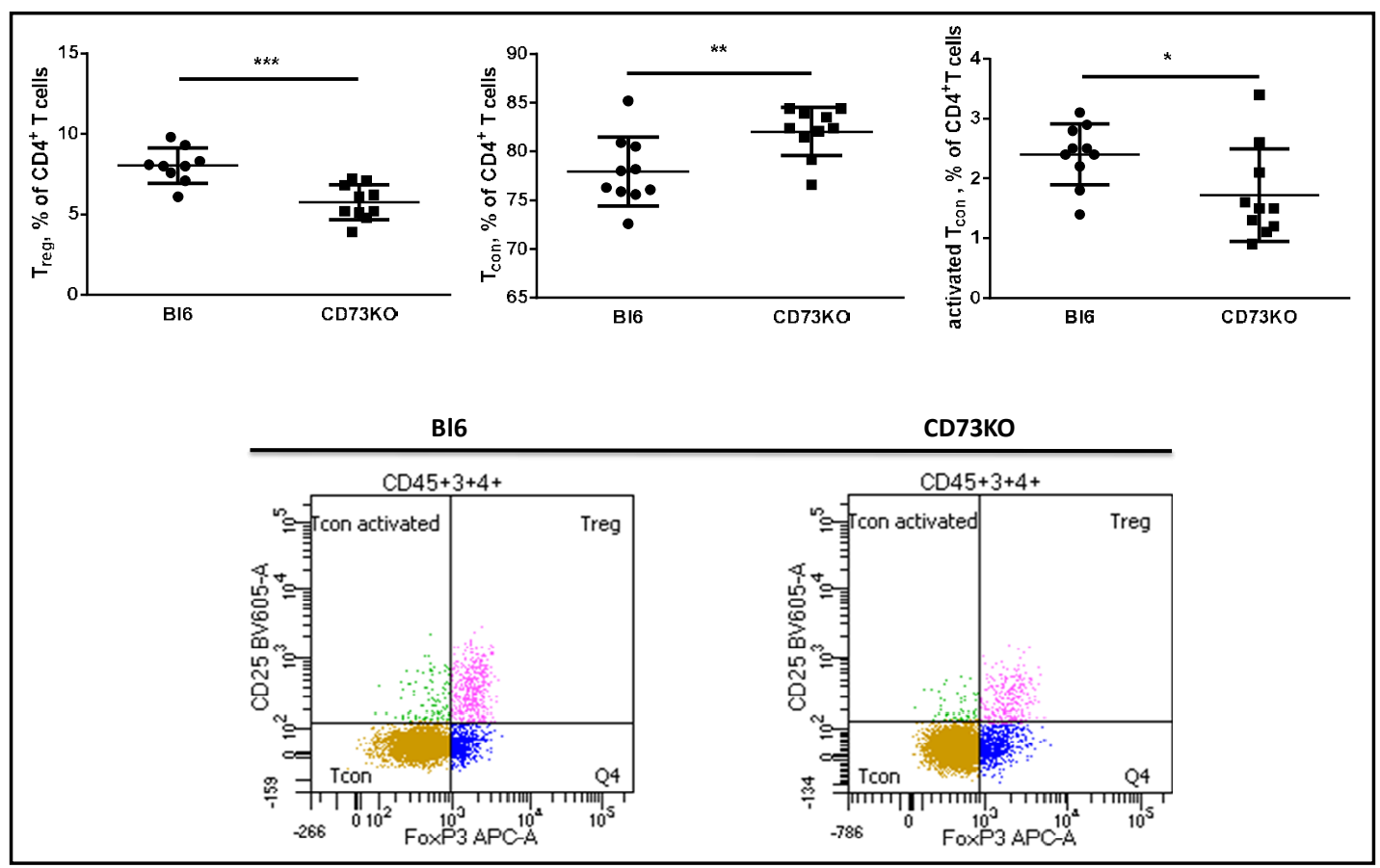

Fig. 4. Influence of CD73-deficience on the Treg, Tcon and activated Tcon in spleens of Bl6 and CD73KO mice. Data are presented as a scatter-plot with mean and SD and analyzed with the unpaired $\mathrm{T}$ test $(* \mathrm{p}<0.05, * *$ $\mathrm{p}<0.01$ and ${ }^{* * *} \mathrm{p}<0.005$ ) - upper panel. Bottom panel - representative FACS pictures from Bl6 vs. CD73KO mice.

\section{Discussion}

The main aim of this work was to characterize immunological cell populations as well as serum cytokine level in CD73KO compared to wild-type healthy mice. Our data demonstrate that these two mouse strains are indeed different in some immunological parameters. This observation is an important one, since CD73 can be recognized as an immune check point molecule [11]. While the KO mice for the well-known immune check point molecule CTLA4 demonstrated a severe phenotype manifesting in an acute and systemic autoimmune pathology [16], the phenotype of knockout mice deficient in CD73 molecule is not affected. So far, the phenotype of mice from this model is rather closer to the mouse model dealing with B7H1 knockout [17]. However, the immunological examination of the healthy CD73 knockout mice is not yet complete, and should be further explored taking into account the importance of the immune check point molecule in context of oncoimmunology [18]. Since, the presence of tumor manipulates drastically the "naïve" immune system of tumor-bearing mice, we want to avoid the comparison of the results from our work with ones obtained from the CD73-inhibiting models of tumor-bearing animals.

Firstlyand importantly, we showed in this study a systemic reduction in IL-6 concentration in serum of CD73-deficient mice. Since IL-6 is a well-known and established marker of acute and chronical inflammation [19], we suggest that the CD73 knockout mice have generally mild inflammation phenotype. Since it has been shown that Ado could increase production of IL-6 through A2A [20], it is tempting to speculate that in the CD73-deficient mice the low level of IL-6 is due to the absence of the Ado catabolism.

Regarding the innate immune system, we found a decrease in amount of NK cells in the CD73-deficient mice. It is surprising because as mentioned before the low amount of Ado (due to CD73 deficiency) should potentially lead to an increase in a frequency of these cells [21]. However, in our recent work with healthy B7H1 knockout mice, we also observed the decrease in NK cells' frequencies in transgenic mice [22]. The exact mechanism leading to 


\section{Cellular Physiology Cell Physiol Biochem 2019;52:1193-1202

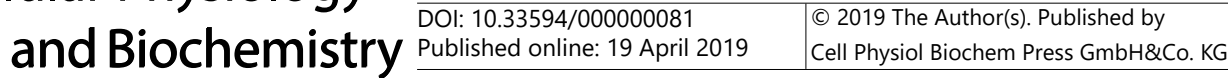 \\ Adam et al.: Immunological Effects of CD73 Deficiency}

this effect in both models is unclear. One can speculate that this reduction might serve as an equation for reduced regulatory potential due to the lack of immune check point molecules. Concerning another type of innate immune cells - DC, we could not detect any changes in the total amount of $\mathrm{cDC}$ but sow a reduction of $\mathrm{pDC}$ as well as of the expression level of the costimulatory molecules CD80 and CD86 in transgenic mice. The same effects were seen by us in the healthy B7H1 knockout mice [22]. Thus, we suppose that lack of the CD73 molecules may be compensated by appropriate changes in the expression of some stimulators on DC. In opposite to the B7H1 knockout model we did not detected differences in percentages of MDSC in the CD73 knockout mice [22].

Could the CD73-deficience influence adaptive immune system? Beyond of the tendency to lower amount of $\mathrm{T}$ cell and to higher amount of $\mathrm{B}$ cells in spleens of the transgenic mice, which in line with our data from the B7H1KO study [22], we obtained several statistically significant data regarding $\mathrm{CD} 4^{+} \mathrm{T}$ cells. First, the percentage of effector $\mathrm{CD} 4^{+} \mathrm{T}$ cells was higher in a case of CD73-deficience. Second, the percentage of memory CD4 ${ }^{+} \mathrm{T}$ was lower in the spleens of transgenic mice. Accumulating evidence suggested the importance of IL-6 for $\mathrm{CD}^{+} \mathrm{T}$ cell differentiation [23], proposing an influence of this cytokine on memory/ effector phenotype of these T cells [24, 25]. Therefore, our data are in line with the abovementioned research. Besides, our finding is also corroborated with a fact that Ado can modulate differentiation and proliferation of $\mathrm{T}$ cells through the A2A receptors [26]. Thus, we can suggest that in absence of CD73 lower Ado concentration could lead to the immune modulating effects on T cells. Interesting to note, that healthy B7H1KO mice did not show differences between strains in the percentage of effector and memory CD4 ${ }^{+} \mathrm{T}$ cells [22], pointing an immunological distinction of both models.

As mentioned before, the Ado-production is one of the important immunosuppressive mechanisms executed by Treg [9]. These immunosuppressive effects of Ado are attributed to the same A2A receptors [27, 28]. According to this fact, we found a low amount of Treg and high amount of Tcon in the spleens of CD73-deficient mice. It should be stressed, that in opposite to the CD73 model healthy B7H1KO mice showed an increased amount of Treg [22].

As mentioned before CD73 is recognized as an immune check point molecule which can be important for development of new immunotherapeutic strategies against cancer [11]. However, a recent report about the immunosuppressive axis CD73/A2A in diffuse large B-cell lymphoma showed that only CD73 expressed on tumor cells correlates with poor survival of cancer patients [29]. Therefore, CD73 knockout mice from this study can be used for further investigation of this molecule in the tumor context.

\section{Conclusion}

Summarizing, we propose a following immunological scenario due to the CD73 absence: the deactivated A2A receptor leads to diminishing of IL-6 production, which in turn could modulate the Treg. Apart from IL-6, the A2A receptor deactivation diminishes the percentage of Treg and shifts the effector/memory balance of $\mathrm{CD} 4^{+} \mathrm{T}$ cells in the effector direction. Therefore, the CD73-deficiency imparts the animals a special immune phenotype which differs from phenotype of other models with a deficiency in immune check point molecules.

\section{Acknowledgements}

Author contributions: conceptualization - SK and AVB; experiments - TA, AM, OI; methodology - SK and AVB; project administration and resources - PPP, JW and AVB; supervision - AB; writing (original draft) - SK and AVB; writing (review \& editing) - all authors. We thank Ms. Michaela Svihla for her excellent technical assistance and Dr. Mara Taverna for her administrative assistance. 


\section{Cellular Physiology Cell Physiol Biochem 2019;52:1193-1202 \begin{tabular}{c|l} 
DOI: 10.33594/000000081 & (c) 2019 The Author(s). Published by
\end{tabular} and Biochemistry Published online: 19 April $2019 \quad$ Cell Physiol Biochem Press GmbH\&Co. KG \\ Adam et al.: Immunological Effects of CD73 Deficiency}

\section{Disclosure Statement}

The authors declare that there are no conflicts of interest.

\section{References}

- 1 Khakh BS, Burnstock G: The double life of ATP. Sci Am 2009;301:84-90.

- 2 Dou L, Chen YF, Cowan PJ, Chen XP: Extracellular ATP signaling and clinical relevance. Clin Immunol 2018;188:67-73.

- 3 Strater N: Ecto-5'-nucleotidase: Structure function relationships. Purinergic Signal 2006;2:343-350.

- 4 Kordass T, Osen W, Eichmuller SB: Controlling the Immune Suppressor: Transcription Factors and MicroRNAs Regulating CD73/NT5E. Front Immunol 2018;9:813.

5 Yegutkin GG: Nucleotide- and nucleoside-converting ectoenzymes: Important modulators of purinergic signalling cascade. Biochim Biophys Acta 2008;1783:673-694.

6 Stagg J, Smyth MJ: Extracellular adenosine triphosphate and adenosine in cancer. Oncogene 2010;29:53465358.

7 Allard B, Turcotte M, Stagg J: CD73-generated adenosine: orchestrating the tumor-stroma interplay to promote cancer growth. J Biomed Biotechnol 2012;2012:485156.

8 Fredholm BB, Chern Y, Franco R, Sitkovsky M: Aspects of the general biology of adenosine A2A signaling. Prog Neurobiol 2007;83:263-276.

9 Whiteside TL, Mandapathil M, Schuler P: The role of the adenosinergic pathway in immunosuppression mediated by human regulatory T cells (Treg). Curr Med Chem 2011;18:5217-5223.

10 Umansky V, Shevchenko I, Bazhin AV, Utikal J: Extracellular adenosine metabolism in immune cells in melanoma. Cancer Immunol Immunother 2014;63:1073-1080.

- 11 Bazhin AV, Amedei A, Karakhanova S: Editorial: Immune Checkpoint Molecules and Cancer Immunotherapy. Front Immunol 2018;9:2878.

12 Thompson LF, Eltzschig HK, Ibla JC, Van De Wiele CJ, Resta R, Morote-Garcia JC, Colgan SP: Crucial role for ecto-5'-nucleotidase (CD73) in vascular leakage during hypoxia. J Exp Med 2004;200:1395-1405.

13 Karakhanova S, Yang Y, Link J, Soltek S, von Ahn K, Umansky V, Werner J, Bazhin AV: Gender-specific immunological effects of the phosphodiesterase 5 inhibitor sildenafil in healthy mice. Mol Immunol 2013;56:649-659.

- 14 Fritz J, Karakhanova S, Brecht R, Nachtigall I, Werner J, Bazhin AV: In vitro immunomodulatory properties of gemcitabine alone and in combination with interferon-alpha. Immunol Lett 2015;168:111-119.

- 15 Bazhin AV, Yang Y, D’Haese JG, Werner J, Philippov PP, Karakhanova S: The novel mitochondria-targeted antioxidant SkQ1 modulates angiogenesis and inflammatory micromilieu in a murine orthotopic model of pancreatic cancer. Int J Cancer 2016;139:130-139.

- 16 Tivol EA, Borriello F, Schweitzer AN, Lynch WP, Bluestone JA, Sharpe AH: Loss of CTLA-4 leads to massive lymphoproliferation and fatal multiorgan tissue destruction, revealing a critical negative regulatory role of CTLA-4. Immunity 1995;3:541-547.

- 17 Nishimura H, Honjo T: PD-1: an inhibitory immunoreceptor involved in peripheral tolerance. Trends Immunol 2001;22:265-268.

18 Bazhin AV, von Ahn K, Fritz J, Werner J, Karakhanova S: Interferon-alpha Up-Regulates the Expression of PD-L1 Molecules on Immune Cells Through STAT3 and p38 Signaling. Front Immunol 2018;9:2129.

19 Tanaka T, Narazaki M, Kishimoto T: IL-6 in inflammation, immunity, and disease. Cold Spring Harb Perspect Biol 2014;6:a016295.

20 Sun Y, Wu F, Sun F, Huang P: Adenosine promotes IL-6 release in airway epithelia. J Immunol 2008;180:4173-4181.

21 Miller JS, Cervenka T, Lund J, Okazaki IJ, Moss J: Purine metabolites suppress proliferation of human NK cells through a lineage-specific purine receptor. J Immunol 1999;162:7376-7382.

- 22 Bazhin AV, von Ahn K, Maier C, Soltek S, Serba S, Diehl L, Werner J, Karakhanova S: Immunological in vivo effects of B7-H1 deficiency. Immunol Lett 2014;162:273-286.

23 Dienz O, Rincon M: The effects of IL-6 on CD4 T cell responses. Clin Immunol 2009;130:27-33. 


\section{Cellular Physiology and Biochemistry}

Cell Physiol Biochem 2019;52:1193-1202

DOI: 10.33594/000000081

24 Rochman I, Paul WE, Ben-Sasson SZ: IL-6 increases primed cell expansion and survival. J Immunol 2005;174:4761-4767.

25 Longhi MP, Wright K, Lauder SN, Nowell MA, Jones GW, Godkin AJ, Jones SA, Gallimore AM: Interleukin-6 is crucial for recall of influenza-specific memory CD4 T cells. PLoS Pathog 2008;4:e1000006.

- 26 Kobie JJ, Shah PR, Yang L, Rebhahn JA, Fowell DJ, Mosmann TR: T regulatory and primed uncommitted CD4 T cells express CD73, which suppresses effector CD4 T cells by converting 5'-adenosine monophosphate to adenosine. J Immunol 2006;177:6780-6786.

27 Ohta A, Kini R, Ohta A, Subramanian M, Madasu M, Sitkovsky M: The development and immunosuppressive functions of CD4(+) CD25(+) FoxP3(+) regulatory T cells are under influence of the adenosine-A2A adenosine receptor pathway. Front Immunol 2012;3:190.

28 Ohta A, Sitkovsky M: Extracellular adenosine-mediated modulation of regulatory T cells. Front Immunol 2014;5:304.

29 Wang X, Zhang T, Song Z, Li L, Zhang X, Liu J, Liu X, Qiu L, Qian Z, Zhou S, Feng L, Hu G, Meng B, Zhai Q, Ren X, Fu K, Li L, Wang P, Zhang H: Tumor CD73/A2aR adenosine immunosuppressive axis and tumor-infiltrating lymphocytes in diffuse large B-cell lymphoma: correlations with clinicopathological characteristics and clinical outcome. Int J Cancer 2019; DOI:10.1002/ijc.32144. 